\title{
Absceso cerebral.
A propósito de una serie de 30 casos \\ Absceso cerebral.
A propósito de una serie de 30 casos
}

\author{
Alberto Fica C., Patricio Bustos G. y Gonzalo Miranda C.
}

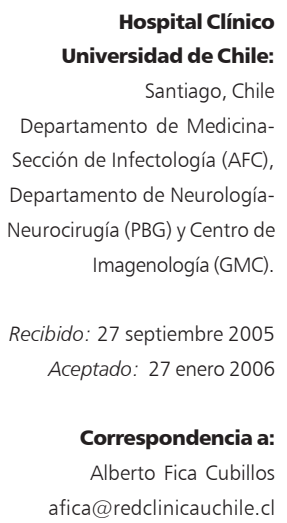

Hospital Clínico

Santiago, Chile Departamento de MedicinaSección de Infectología (AFC), Departamento de NeurologíaNeurocirugía (PBG) y Centro de

Recibido: 27 septiembre 2005 Correspondencia a: afica@redclinicauchile.cl

\section{Brain abscess. About a clinical series of 30 patients}

A retrospective study was designed to ascertain the main clinical features of 30 patients admitted for brain abscess between 1989 and 2005. A contiguous source of infection was identified in $40 \%$ of cases, direct inoculation secondary to trauma or neurosurgery in $23,3 \%$, and a distant source in $23,3 \%$. Fever, headache and sensorial involvement were the main clinical manifestations. Temporal and frontal lesions were predominant. Microbiological information was available in only half of cases, prevailing polymicrobial and grampositive bacterial infections. Most patients underwent surgical procedures $(66.7 \%)$ and the rest received only medical therapy. During antimicrobial treatment, 6 patients $(20 \%)$ suffered a serious adverse event that prompted discontinuation or change of therapy. Failure of treatment was observed in $40 \%$, including 3 deaths $(10 \%)$, non-programmed surgical drainage or reintervention $(16.7 \%)$ and changes of the antimicrobial regimen (13.3\%). Eight patients were discharged with sequels $(26.7 \%)$, and during follow-up, $21 \%$ presented major events (death or brain abscess-related readmission).

Key words: Brain abscess, central nervous system infections, diagnosis, therapy, outcome.

Palabras claves: absceso cerebral, infecciones del sistema nervioso central, tratamiento, evolución.

\section{Introducción}

$\mathrm{E}$ 1 absceso cerebral corresponde a una colección localizada de pus en el parénquima cerebral. Es una patología infrecuente pero de una evolución prolongada y que gracias a los avances en imagenología, técnicas quirúrgicas y terapia antimicrobiana, sólo ocasionalmente produce la muerte.

Las manifestaciones clínicas son variadas, a veces de instalación violenta, y no siempre se encuentra presente la tríada característica de fiebre, cefalea y signos focales. El diagnóstico diferencial es amplio y la sospecha clínica debe ser precoz para lograr un tratamiento oportuno, multidisciplinario y que asegure un buen pronóstico.

Las causas y el manejo de esta patología han experimentado cambios importantes en las últimas décadas y las más recientes series clínicas nacionales publicadas datan de la década de $1980^{1-3}$. Por ejemplo, se ha reportado que la fuente neuro-quirúrgica se ha incrementado como causa de estos abscesos, con disminución del foco ótico, y que los tratamientos basados en el uso de cloranfenicol y penicilina se han abandonado hacia una terapia basada en cefalosporinas de tercera generación ${ }^{4,5}$. De la misma manera el advenimiento de la cirugía estereotáxica y la resonan- cia magnética (RM) han modificado la aproximación diagnóstica y terapéutica de estos abscesos ${ }^{4}$.

Estos antecedentes hacen necesario efectuar una revisión actualizada de esta patología que permita graficar cómo la mortalidad o las secuelas se han reducido, las fuentes de origen han cambiado y que el enfoque terapéutico, ya sea médico o quirúrgico, también se ha modificado.

\section{Metodología}

Se efectuó una búsqueda retrospectiva de todos los egresos hospitalarios atendidos en el Hospital Clínico de la Universidad de Chile y codificados como absceso cerebral entre 1987 y mayo del 2005. Para ello se utilizaron los códigos 324.0 (hasta el año 2001) y luego el G06.0 (desde 2002) de acuerdo a la Clasificación Estadística Internacional de Enfermedades y Problemas relacionados con la Salud (CIE 10, décima revisión. OPS Publicación científica n ${ }^{\circ}$ 554, vol 3 1995).

Debido a lo infrecuente de esta patología, todos los casos fueron considerados para análisis, incluyendo los pacientes trasladados desde otros servicios u hospitales o reingresos por complicaciones neuro-quirúrgicas. 
Los casos fueron revisados de acuerdo a una pauta estructurada que incluyó datos demográficos, antecedentes mórbidos, síntomas y su inicio, fecha de hospitalización y egreso, datos básicos de laboratorio, estudio por imágenes, resultados microbiológicos y fuente probable de infección, localización del absceso, tipo de tratamiento antimicrobiano y su extensión, intervención quirúrgica, uso de corticosteroides, condición clínica al egreso y secuelas.

El tratamiento antimicrobiano fue clasificado de acuerdo al uso de compuestos anti-anaerobios, penicilina o ampicilina, cefalosporinas de tercera generación y tipo de terapia anti-estafilocóccica. Fue registrada la fecha de inicio y término del esquema antimicrobiano para calcular la extensión del tratamiento. En aquellos casos en que el absceso cerebral apareció como una complicación de reconocimiento tardío y con uso previo de antimicrobianos por otras razones, se consideró como fecha de inicio de esta patología, aquella correspondiente al momento del diagnóstico.

Definiciones. Fracaso del tratamiento médico o quirúrgico fue definido como el deceso del paciente, la necesidad de drenar el absceso cerebral a pesar del tratamiento antimicrobiano previo, una segunda intervención quirúrgica del absceso o cambio de espectro del tratamiento antimicrobiano dirigido al manejo del absceso o de los focos de entrada por cobertura insuficiente.

Se efectuó una comparación estadística para determinar si la evolución del paciente o la letalidad estuvo asociada a la edad, presencia de diabetes mellitus, patología tumoral o inmunosupresión, manifestaciones clínicas, puerta de entrada, localización del absceso, microbiología o modalidad de tratamiento. Para ello se efectuaron tablas de contingencia con la prueba bilateral de Fisher.

Se consignó el seguimiento post-egreso en los pacientes para identificar muerte o rehospitalización por causas relacionadas. Para los cálculos de sobrevida se consideraron como eventos la muerte durante la hospitalización o en el seguimiento y la rehospitalización por una causa relacionada (recidiva del tumor de base, fístula LCR o convulsiones). Se consideró arbitrariamente un año como el tiempo adecuado de seguimiento post-egreso. Los pacientes que no cumplieron este criterio fueron considerados perdidos.

\section{Resultados}

Población identificada. Un total de 30 casos pudo ser identificado en el período 1987 hasta mayo del 2005. Ocho fichas de pacientes afectados en 1989 o antes, no pudieron ser rescatadas y no fueron incluidas en este estudio. La mayor parte de los casos de esta serie $(n=21 ; 70 \%)$ fue confirmada por biopsia compatible, hallazgos quirúrgicos, tinción de Gram directa o cultivo de la lesión. En 2 de estos 21 pacientes el diagnóstico fue planteado exclusivamente durante la biopsia estereotáxica, realizada ante la sospecha de un tumor cerebral. En 8 pacientes $(26,7 \%)$ el diagnóstico fue confirmado por imágenes compatibles con un absceso cerebral y una recuperación clínica y radiológica asociada al tratamiento antimicrobiano. En un caso en el cual no hubo recuperación, el diagnóstico fue confirmado por imágenes asociadas a un foco de entrada adyacente $(3,3 \%)$.

Datos biodemográficos. Se observó un discreto predominio de casos de sexo masculino $(\mathrm{n}=18 ; 60 \%)$ y la edad promedio fue de 43,7 años (rango 16 a 77 años).

Factores de riesgo. Casi un tercio de los pacientes presentaba una intervención neuro-quirúrgica previa $(n=8 ; 26,7 \%)$. Cuatro de estos casos estaban asociados a tumores antiguos o recientes del SNC, 2 casos a patología vascular cerebral y 2 a TEC. Otros factores de riesgo identificados en esta población fueron otitis media crónica $(\mathrm{n}=5,16,7 \%)$ y diabetes mellitus o inmunosupresión, incluyendo un caso de leucemia mieloide aguda $(n=4,13,3 \%)$. Además, otros 2 casos presentaban antecedentes de cardiopatía congénita (uno de ellas corregida), uno de enfermedad de depósito (enfermedad de Gaucher) y otro de cáncer epidermoide del seno maxilar derecho tratado con cirugía y radioterapia muchos años antes (Tabla 1). En $30 \%$ de los casos no se identificó factores de riesgo.

Puerta de entrada. Pudo ser reconocida en la mayor parte de los casos (26 de 30 casos, 86,6\%, Tabla 2). La más frecuente correspondió a un foco ótico ( 7 casos; $23,3 \%$ del grupo total), secundado por procedimientos neuro-quirúrgicos en 5 casos $(16,7 \%)$. Otros focos observados correspondieron a traumatismo o foco sinusal con 3 casos (10\%) cada uno, foco cardíaco, dental o pulmonar con 2 casos $(6,7 \%)$ cada uno y, en forma más infrecuente, foco abdominal o fístula de LCR (no asociada a neurocirugía o trauma) con un caso $(3,3 \%)$ cada uno. (Tabla 2) Desde el punto de vista patogénico, una lesión por contigüidad fue identificada en 11 casos $(36,7 \%)$, especialmente por un foco sinusal $u$ ótico $(n=10,33,3 \%)$. Una puerta de entrada post-quirúrgica o post-traumática fue registrada en 8 casos $(26,7 \%)$ y una fuente distante con diseminación hematógena en otros 7 casos (dental, cardíaco, pulmonar o abdominal; 23,3\%). La fuente cardíaca estuvo asociada a un caso de endocarditis infecciosa causada por Staphylococcus aureus, y el otro caso a una comunicación interauricular con cortocircuito de derecha a izquierda. Por su parte, la puerta de entrada pulmonar se asoció a un caso de neumonía y en otro con una malformación arterio-venosa. 
Tabla 1. Parámetros demográficos, factores de riesgo y puerta de entrada en 30 pacientes con absceso cerebral

\section{Parámetro \\ Datos biodemográficos}

Distribución por sexo (M/F)

Edad (años)

Factores de riesgo*

Con intervención neuro-quirúrgica previa

- Tumores del SNC

- Patología vascular

- TEC

- Subtotal

Frecuencia o valor promedio

$\begin{array}{cc}18 / 12 & 60 / 40 \\ 43,7 & 16-77\end{array}$

Rango o \%

$16-77$
Manifestaciones clínicas. Fiebre fue la más frecuente, secundada por cefalea y luego focalización neurológica o compromiso de conciencia (casi la mitad de la serie), aunque sólo $20 \%$ de los casos presentó la tríada clásica de cefalea, fiebre y focalización. Las convulsiones antes del ingreso fueron infrecuentes en esta serie $(10 \%)$ y sólo un tercio presentó signos meníngeos al examen físico (Tabla 3). No se pudo establecer una escala de ingreso sobre el nivel de conciencia por falta de datos estandarizados o comentarios en la ficha clínica.

Las manifestaciones focales incluyeron déficit motor parcial o plejía $(\mathrm{n}=10)$, afasia $(\mathrm{n}=2)$ y compromisos de pares craneanos del VI o VII par $(n=2)$. Un caso se presentó con hemiparesia y afasia combinada.

Sólo 2 pacientes presentaron manifestaciones cerebelosas que incluyeron ataxia, vértigo, disdiadococinesia, dismetría o nistagmus.

En 23 casos con información precisa sobre el inicio de los síntomas y cuyos cuadros no se presentaron como complicación intrahospitalaria, se pudo efectuar un análisis sobre la duración de ellos. Aproximadamente la mitad de los casos se hospitalizó por un cuadro de $<5$ días de evolución y dos tercios por un cuadro no superior a 10 días (Tabla 3).

Laboratorio. El valor promedio del recuento de leucocitos fue de $15.521 / \mathrm{mm}^{3}$ aunque $56,7 \%$ presentaba valores superiores al rango normal. La VHS también demostró un alza moderada (Tabla 3).

Localización. La más frecuente fue en el lóbulo temporal en 11 casos (36,7\%), secundada por abscesos en el lóbulo frontal en 9 casos (30\%) (Tabla 4). En aquellos casos donde el foco de infección fue ótico, siempre el absceso estuvo ligado al cerebelo o al lóbulo temporal (4 temporales y 3 cerebelosos). Cinco pacientes presentaron abscesos múltiples $(16,7 \%)$.

Imagenología. La mayor parte de los casos fueron estudiados con TAC cerebral $(n=25,83,3 \%)$ y una minoría con $\mathrm{RM}(\mathrm{n}=3,10 \%)$. En 2 casos se realizó un estudio mixto con ambas técnicas $(6,7 \%)$. En ninguno de los casos se efectuó estudios con técnicas de medicina nuclear o arteriografía cerebral. En 23 de los 25 pacientes estudiados con TAC se pudo rescatar, con algún grado de detalle, el informe radiológico. Éste señaló 2 casos de cerebritis $(8,7 \%)$ y el resto alguna forma concordante con absceso cerebral ("absceso" en 12 casos $(52,2 \%)$, "lesión hipodensa con refuerzo al medio de contraste" en 8 casos $(34,8 \%)$ e "imagen loculada" en el caso restante $(4,3 \%))$. Los 2 casos sospechosos de tumor del SNC fueron informados en los pacientes estudiados por RM.

Microorganismos identificados. En la mitad de los casos se identificaron microorganismos causales, ya sea por la tinción de Gram directa del material extraído 
o por cultivos $(\mathrm{n}=15,50 \%)$ (Tabla 5). El agente etiológico fue identificado en muestras directas del absceso cerebral en 14 de 20 pacientes operados. El otro caso fue diagnosticado mediante hemocultivos en un paciente con endocarditis infecciosa causada por $S$. aureus. En 7 de los 15 casos hubo desarrollo de microorganismos en los cultivos (46,6\%). En los 8 casos restantes, sólo hubo información de la tinción de Gram directa.

Los hallazgos más frecuentes correspondieron a evidencias de infección polimicrobiana o a infección por microorganismos grampositivos en los cuales no hubo desarrollo posterior ( $\mathrm{n}=5 ; 33,3 \%$ cada grupo).

En 4 casos se identificó el género Staphylococcus, ya fuera en cultivo mono o polimicrobiano. Tres de estos correspondían a $S$. aureus y la mitad de los 4 aislamientos era resistente a cloxacilina. No se encontró ninguna asociación significativa entre agente y puerta de entrada. En esta serie se identificó Pseudomonas aeruginosa en un caso, correspondiente a un paciente joven con puerta de entrada ótica y sin un perfil de multiresistencia ni coexistencia con diabetes mellitus.

Tratamiento. El tratamiento quirúrgico del absceso o de la hipertensión endocraneana fue aplicado en 20 pacientes $(66,7 \%)$, incluyendo 14 casos con vaciamiento o drenaje del absceso, uno con drenaje ventricular-externo (DVE) aislado y 5 casos con ambos procedimientos (Tabla 6). La cirugía se desarrolló en promedio a los 12 días del ingreso (rango: -4 a 52 días); la cifra con valor negativo corresponde a un paciente que fuera trasladado a nuestra institución después de ser operado en otro recinto. La mayor parte de los pacientes fue intervenida quirúrgicamente durante los primeros 10 días de ingreso (12 de $20 ; 60 \%$ ) y $85 \%$ antes de los 20 días. Seis pacientes (30\%) requirieron dos o más intervenciones quirúrgicas para el vaciamiento del absceso o para la instalación o retiro de drenajes ventriculares externos o internos (rango 2 a 4 operaciones). En 4 de estos 20 casos se efectuó cirugía estereotáxica $(20 \%)$.

Seis pacientes en la serie fueron sometidos a procedimientos de derivación del LCR (DVE, 20\%) y 5 a cirugía radical de oído o drenaje de absceso retroauricular (16,7\%, Tabla 6). Casi la mitad de la serie recibió corticosteroides como parte del tratamiento $(n=13$; 43,3\%).

Excluyendo a los 2 pacientes que fueron operados bajo la sospecha de un tumor cerebral, se aplicó tratamiento médico en 8 pacientes de la serie $(26,7 \%)$. Las razones para seleccionar esta modalidad de tratamiento fueron precisadas en 6 de estos 8 casos. En 2 de ellos, la cirugía no se realizó por trombocitopenia, en 3 casos por lesiones pequeñas o sin efecto de masa y en
Tabla 3. Manifestaciones clínicas, duración de síntomas y laboratorio en 30 pacientes con absceso cerebral

\begin{tabular}{|c|c|c|}
\hline Manifestaciones clínicas & $\mathbf{n}$ & $\%$ \\
\hline Fiebre & 22 & 73,3 \\
\hline Cefalea & $17 * *$ & 58,6 \\
\hline Focalización neurológica & 14 & 46,7 \\
\hline Fiebre + cefalea + focalización neurológica & 6 & 20 \\
\hline Compromiso de conciencia & 14 & 46,7 \\
\hline Convulsiones & 3 & 10 \\
\hline Manifestaciones cerebelosas & 2 & 6,7 \\
\hline Signos meníngeos & 9 & 30 \\
\hline \multicolumn{3}{|l|}{ Duración de síntomas antes del ingreso } \\
\hline 0 a 5 días & 11 & 47,8 \\
\hline 6 a 10 días & 4 & 17,4 \\
\hline 11 a 15 días & 2 & 8,7 \\
\hline 16 a 20 días & 2 & 8,7 \\
\hline$>20$ días & 4 & 17,4 \\
\hline \multicolumn{3}{|l|}{ Laboratorio } \\
\hline Leucocitos $/ \mathrm{mm}^{3} * * *$ (promedio, rango) & 10.521 & 3.000 a 26.600 \\
\hline Leucocitos $>10000 / \mathrm{mm}^{3}$ & 17 & 56,7 \\
\hline VHS mm/hr **** (promedio, rango) & 45 & 2 a 123 \\
\hline
\end{tabular}

Tabla 4. Localización anatómica de abscesos cerebrales en $\mathbf{3 0}$ pacientes

\begin{tabular}{|lcc|}
\hline Localización & Frecuencia & \% \\
\hline Temporal & 11 & 36,7 \\
\hline Frontal & 9 & 30 \\
\hline Cerebelosa & 3 & 10 \\
\hline Parietal & 3 & 10 \\
\hline Occipital & 1 & 3,3 \\
\hline Profunda & 2 & 6,7 \\
\hline No precisado & 1 & 3,3 \\
\hline Total & 30 & 100 \\
\hline
\end{tabular}

Nota: la localización fue asignada a la región anatómica predominante 
un caso estaba contraindicada por cardiopatía coronaria.

Diferentes esquemas antimicrobianos fueron utilizados para el manejo de estos pacientes y la distribución se muestra en la Tabla 7. Las cefalosporinas de tercera generación en asociación con compuestos con actividad anti-anaeróbica, fueron los esquemas más utilizados. El tratamiento anti-infeccioso tuvo una extensión promedio de 39,8 días (rango: 5-114 días) y $90 \%$ de los pacientes recibió tratamiento por 3 o más semanas. Los pacientes restantes recibieron tratamien-

Tabla 5. Agentes etiológicos detectados en 15 pacientes con abscesos cerebrales e información disponible

\begin{tabular}{lcc} 
Condición & Frecuencia & $\%$ \\
Gram o cultivo polimicrobiano* & & \\
Staphylococcus coagulasa negativa + Klebsiella pneumoniae & 1 & 6,7 \\
Pseudomonas aeruginosa y anaerobios & 1 & 6,7 \\
Staphylococcus aureus + Klebsiella pneumoniae & 1 & 6,7 \\
Gram polimicrobiano & 2 & 13.3 \\
Subtotal & 5 & 33,3 \\
Infección monomicrobiana & 5 & 33,3 \\
Cocáceas grampositivas al Gram (sin desarrollo posterior) & 5 \\
Staphylococcus aureus sin otro agente asociado & 2 & 13,3 \\
Escherichia coli & 1 & 6,7 \\
Bacilos gramnegativos al Gram & 1 & 6,7 \\
Anaerobios estrictos sin otras especies** & 1 & 6,7 \\
Subtotal & 10 & 66,6 \\
Total & 15 & 100 \\
\hline
\end{tabular}

\begin{tabular}{|lcc|}
\hline $\begin{array}{l}\text { Tabla 6. Detalles del tratamiento médico y/o quirúrgico en } \mathbf{3 0} \text { pacientes } \\
\text { con absceso cerebral }\end{array}$ & Frecuencia & $\%$ \\
\hline $\begin{array}{l}\text { Parámetro } \\
\text { Tratamiento quirúrgico }\end{array}$ & \\
\hline Intervenciones sobre el SNC & 14 & 46,7 \\
\hline - Drenaje o vaciamiento absceso & 1 & 3,3 \\
- DVE & 5 & 16,7 \\
- Drenaje + DVE & 20 & 66,7 \\
- Total & 5 & 16,7 \\
Cirugía sobre foco ótico* & 8 & 26,7 \\
\hline Tratamiento médico & 2 & 6,7 \\
\hline Tratados o estudiados inicialmente como & & \\
tumor del SNC & & \\
\hline *: No excluyente sobre otro tipo de cirugía; DVE: derivación ventricular externa. & \\
\hline
\end{tabular}

to por 5, 11 y 16 días respectivamente, en el primer caso por deceso del paciente. No se especificó en la ficha clínica la razón por la cual los otros dos pacientes fueron tratados en forma abreviada (11 y 16 días), aunque ambos de ellos egresaron en buenas condiciones y la lesión era única.

El 20\% de los pacientes $(n=6)$ presentó alergia o reacciones adversas a compuestos $\beta$-lactámicos, vancomicina o ciprofloxacina, lo que obligó a suspender o modificar el esquema terapéutico. Éstas incluyeron rash o neutropenia y en ningún caso se asociaron a manifestaciones anafilácticas. La alergia a compuestos $\beta$-lactámicos incluyó penicilina o cloxacilina y cefalosporinas en 5 de los 6 casos. En un caso se presentó una neutropenia secundaria posiblemente a vancomicina y posteriormente alergia cutánea a ciprofloxacina. En 3 de los 5 casos con alergia a $\beta$ lactámicos se sospechó también la participación de anti-convulsivantes en la génesis del rash.

La duración de la hospitalización tuvo una extensión promedio de 43,5 días con un rango de 6 a 127 días.

Evolución. De acuerdo a las definiciones operacionales entregadas, se observó un fracaso del tratamiento médico o quirúrgico en un elevado porcentaje de los pacientes $(\mathrm{n}=12,40 \%)$. De estos 12 pacientes, 3 fallecieron $(25 \%), 5$ requirieron una nueva intervención quirúrgica $(41,7 \%)$ y 4 un ajuste o cambio de antimicrobianos $(33,3 \%)$.

La mortalidad en esta serie fue de $10 \%(n=3)$. De los pacientes que sobrevivieron, un cuarto de ellos $(\mathrm{n}=8$; $26,7 \%$ ) quedó con secuelas manifiestas al momento del egreso o durante el seguimiento. Éstas incluyeron un daño neurológico significativo que impedía la movilización ( 2 pacientes), disartria o paresia leve (2 pacientes), frontalización, hemiparesia, fístula de LCR y alteraciones de memoria de carácter menor (en el resto de los casos, respectivamente). La suma de pacientes

\begin{tabular}{|c|c|c|}
\hline Antimicrobiano & Frecuencia & $\%$ \\
\hline Penicilina o ampicilina & 14 & 46,7 \\
\hline Cefalosporinas de $3^{\mathrm{a}}$ generación & 25 & 83,3 \\
\hline Carbapenémicos & 3 & 10 \\
\hline Cloxacilina o vancomicina & 16 & 53,3 \\
\hline Metronidazol o cloranfenicol & 25 & 83,3 \\
\hline
\end{tabular}


que fallecieron o quedaron con algún grado de déficit alcanzó a $36,7 \%$ de la serie $(\mathrm{n}=11)$.

No se identificaron factores asociados a letalidad o secuelas al alta, tales como edad del paciente, tipo de síntomas al ingreso, diabetes mellitus o inmunosupresión, patología tumoral del SNC, localización del absceso, microorganismo identificado o intervenciones quirúrgicas. La letalidad del grupo tratado en forma médica fue similar a la del grupo quirúrgico $(10 \%$ vs $12,5 \%$ ).

Seguimiento. Se contó con esta información en 19 de los 27 sobrevivientes $(70,4 \%)$, con un seguimiento promedio de 135 días; sin embargo, no fue mayor a 3 meses para la mayor parte de los casos (12 pacientes, $63,2 \%$ ). Sólo 5 pacientes contaron con un seguimiento hasta un año post-egreso $(26,3 \%)$. Durante este seguimiento, 3 pacientes se rehospitalizaron por una causa relacionada (fístula de LCR, crisis convulsiva y reconocimiento tardío de un tumor en el sitio del absceso, respectivamente); un cuarto paciente falleció por recidiva tumoral en su domicilio. Este grupo representa el $21 \%$ del total en seguimiento y los eventos ocurrieron antes de los 2 meses post-alta. La Figura 1 representa los eventos de muerte o rehospitalizaciones por causas relacionadas ocurridas desde el ingreso. Se puede apreciar que la curva sólo se estabiliza desde el cuarto mes desde la fecha de ingreso.

\section{Discusión}

El reducido número de casos reunidos en este trabajo, revela las dificultades para obtener un conjunto significativo de pacientes con absceso cerebral, a pesar de utilizar amplias ventanas de tiempo. Sin embargo, nuestros resultados están en concordancia con la literatura respecto a la diversidad de condiciones comórbidas y puertas de entrada, a la distribución clínica pleomórfica que sólo ocasionalmente se presenta como una tríada clásica, a los hallazgos microbiológicos, al uso de diversas estrategias diagnósticas y terapéuticas y a la baja letalidad observada.

Factores de riesgo y puerta de entrada. Los factores de riesgo identificados demostraron un amplio abanico de condiciones, con el predominio de intervenciones neuro-quirúgicas, de otitis media crónica y de condiciones mórbidas sistémicas tales como diabetes mellitus, inmunosupresión, enfermedad de depósito y leucemia. Estos factores participan facilitando una infección por inoculación directa, por contigüidad o diseminación hematógena. Las cardiopatías congénitas que habitualmente participan en abscesos cerebrales, corresponden a defectos de pared con diseminaciones hematógenas paradójicas con cortocircuito de dere-

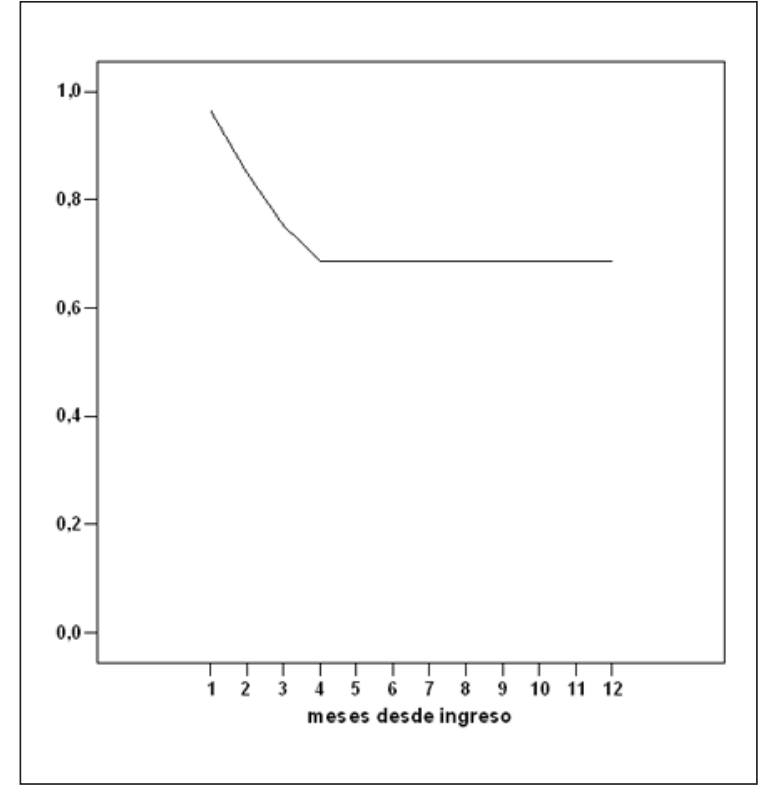

cha a izquierda. Uno de los casos observados en esta serie mantenía un defecto activo y otro había sido reparado varios años antes.

La puerta de entrada predominante fue por contigüidad, especialmente ligada a un proceso ótico o sinusal. El absceso cerebral otogénico se presenta en niños o pacientes jóvenes, portadores de otitis media crónica y característicamente se ubica en el lóbulo temporal o en el cerebelo ${ }^{3,6,7}$. La frecuencia de abscesos otogénicos tiende a disminuir en las series más recientes y ha sido explicado por un mejor control sobre la otitis media crónica ${ }^{5}$. El absceso cerebral es una complicación infecciosa intracraneal infrecuente en pacientes con sinusitis bacteriana y cuando ello ocurre se asocia característicamente a pansinusitis ${ }^{8}$.

La segunda fuente más importante de abscesos cerebrales estuvo ligada a procedimientos neuro-quirúrgicos o post-traumáticos. A pesar de que se ha señalado un incremento de los abscesos ligados a procedimientos neuro-quirúrgicos en los últimos años, este perfil no fue observado en nuestros pacientes. De acuerdo a datos nacionales, el absceso cerebral es una rara complicación infecciosa post-quirúrgica, que no supera un caso por cada 1.000 pacientes operados ${ }^{9}$.

La diseminación hematógena desde diferentes focos ocupó una fracción importante de casos en esta serie. Las limitaciones a la penetración de microorganismos que impone la barrera hemato-encefálica, explica el bajo porcentaje observado de abscesos cerebrales en pacientes con endocarditis infecciosa, fenómeno que no supera el 1 a $4 \%{ }^{10}$. Las complicaciones
Figura 1. Tabla de sobrevida libre de eventos desde el ingreso (muertes o rehospitalizaciones relacionadas) en 30 casos de absceso cerebral. El eje vertical denota la fracción de pacientes sin eventos. 
neurológicas, vasculares o infecciosas son frecuentes en pacientes con malformaciones arteriovenosas pulmonares, tal como fue observado en un caso con esta condición en nuestra serie ${ }^{11}$.

Manifestaciones clínicas. La triada característica asociada al absceso cerebral es una manifestación infrecuente en esta patología y por ello la sospecha clínica sigue siendo el pilar diagnóstico. Los síntomas cerebelosos habitualmente se presentan en pacientes con antecedentes de otitis media crónica y facilitan el diagnóstico. El corto tiempo de evolución antes del ingreso podría explicar la baja frecuencia de convulsiones en estos pacientes.

Estudio de imágenes. Los estudios imagenológicos reflejan el estadio histopatológico del absceso cerebral, de ahí que los hallazgos varían si lo observamos en etapa de cerebritis o de encapsulamiento.

En la etapa inicial de cerebritis, la TAC muestra una lesión hipodensa mal definida que, tras la administración de medio de contraste, presenta un realce en anillo. En la etapa con cápsula se puede visualizar un centro más hipodenso, que refleja la necrosis central con licuefacción, rodeada por un anillo de mayor densidad correspondiente a la cápsula, que se realza tras la administración de medio de contraste. En ambos casos en una TAC se observa edema vasogénico que rodea a la lesión hipodensa. El diagnóstico en etapa de cerebritis es dificultoso y por esto rara vez se diagnostica, hecho concordante con lo encontrado en esta serie $^{12}$.

Los hallazgos en RM con secuencias clásicas, asociados a un estudio de difusión, son bastante característicos para establecer el diagnóstico. En etapa de cerebritis se observa una imagen hipointensa en T1 e hiperintensa en T2 con intensificación en anillo. En las secuencias clásicas y en etapa de encapsulamiento se demuestra un centro hipointenso en $\mathrm{T} 1$ e hiperintenso en T2 con una cápsula isointensa a moderadamente hiperintensa en T1 e hiperintensa en T2. Tras la inyección de gadolinio, la cápsula muestra un realce en anillo con bordes bien definidos ${ }^{13}$. Los estudios de difusión han permitido incrementar la especificidad de la RM, al discriminar la naturaleza de la colección al interior del absceso, demostrando una hiperintensidad central $^{14,15}$.

El diagnóstico diferencial por imágenes comprende principalmente neoplasias primarias y secundarias con necrosis central, y en ocasiones infarto, enfermedad desmielinizante y necrosis por radiación ${ }^{16}$. La combinación de los hallazgos en las secuencias clásicas, más el estudio de difusión y últimamente el uso de la espectroscopia por RM, permiten establecer el diagnóstico con un alto grado de confianza. En TAC el diagnóstico es más difícil por lo que actualmente se prefiere el estudio por $\mathrm{RM}^{14,15}$. Concordante con esta limitación, en 2 pacientes de esta serie se había planteado el diagnóstico de tumor del SNC.

Localización. El predominio de lesiones en lóbulos frontales y temporales, como en esta serie, es una característica concordante con la experiencia mundial y naciona $^{17,18}$. Esta distribución responde al predominio de lesiones por contigüidad como mecanismo patogénico.

Estudio microbiológico y etiología. Diversas especies de bacterias aeróbicas y anaeróbicas estrictas aparecen involucradas en un absceso cerebral. Éstas pueden presentarse como infecciones mono o polimicrobianas. Los microorganismos recuperados dependen de la puerta de entrada y su distribución es estereotipada. En las lesiones por contigüidad, predominan las bacterias grampositivas aerobias y anaerobias estrictas (Streptococcus grupo milleri y Peptostreptococcus) y luego bacilos gramnegativos anaerobios tales como Fusobacterium y Bacteroides; bacilos grampositivos anaerobios (Clostridium) y Peptococcus. Cuando la puerta de entrada es ótica, se describe un claro predominio de Bacteroides sp (incluso B. fragilis), y de Proteus sp. En el caso de los abscesos post-quirúrgicos o post-traumáticos, ellos están asociados fundamentalmente a $S$. aureus y en el caso de focos a distancia, la microbiología refleja la puerta de entrada, como por ejemplo Streptococcus grupo viridans o $S$. aureus en el caso de endocarditis infecciosa ${ }^{4,9,16,17,19,20}$.

Las especies identificadas en nuestro trabajo fueron similares a las señaladas en la literatura médica, en concordancia con la puerta de entrada. No obstante, el rendimiento del cultivo o de la tinción de Gram directa fue bajo $(50 \%)$ impidiendo un diagnóstico microbiológico con exactitud. Las causas de este bajo rendimiento microbiológico no pudieron ser esclarecidas pero parecen ligadas a una baja solicitud de cultivos, tal como fue observado en el mismo centro para pacientes afectados con espondilodiscitis, y probablemente a la omisión frecuente de estudio para bacterias anaerobias estrictas $^{21}$.

En esta serie no se identificaron abscesos asociados a infecciones fúngicas como Aspergillus sp, Toxoplasma gondii o Nocardia sp, tal como ha sido descrito en diferentes reportes por autores naciona$\mathrm{les}^{22-24}$. Ello se explica porque no hubo pacientes con trasplante de precursores hematopoyéticos, de órganos sólidos o con SIDA en la serie y porque la toxoplasmosis cerebral se maneja habitualmente con tratamiento médico empírico en un contexto clínico compatible.

Tratamiento. El tratamiento de esta patología requiere un manejo médico-quirúrgico en la mayor parte de los casos. Además de su utilidad diagnóstica, el 
tratamiento quirúrgico está especialmente indicado en pacientes con abscesos de diámetro mayor $(>2-3 \mathrm{~cm})$, aquellos con un importante efecto de masa o ante fracasos del tratamiento médico ${ }^{4,16}$. El tratamiento médico con antimicrobianos empíricos adecuadamente seleccionados, puede ser instituido ante la presencia de colecciones pequeñas, lesiones múltiples o profundas o aquellas asociadas a meningitis ${ }^{16}$. En estos casos se requiere de un seguimiento periódico con TAC o RM cerebral.

El tratamiento quirúrgico puede ser realizado mediante una trepanación mínima, craneotomía o por cirugía estereotáxica. Esta última representa un procedimiento invasor de menor morbilidad postoperatoria y que puede ser utilizado con fines diagnósticos o terapéuticos. Los procedimientos quirúrgicos pueden estar destinados al vaciamiento o escisión del absceso, a la instalación de un drenaje ventricular externo o al control de la puerta de entrada, especialmente ótica o sinusal.

Las cefalosporinas de tercera generación y el metronidazol poseen un espectro antimicrobiano y una penetración al SNC adecuados, útil para la mayor parte de los microorganismos asociados a esta patología. Este esquema es muy utilizado en forma empírica debido a la naturaleza polimicrobiana de las infecciones asociadas a un foco ótico o dental y a sinusitis, las que constituyeron un porcentaje importante en esta serie. Los esquemas para infecciones neuro-quirúrgicas deben considerar una cobertura adecuada para $S$. aureus y además la posibilidad de resistencia a cloxacilina, un problema emergente a lo largo de este estudio. En nuestros pacientes no pudimos relacionar el fracaso del tratamiento médico-quirúrgico a algún factor o esquema específico.

Un porcentaje significativo de pacientes (20\%) experimentó reacciones adversas durante el tratamiento antimicrobiano, que obligó a su suspensión y reemplazo. Los $\beta$-lactámicos estuvieron involucrados en la mayor parte de los casos, aunque la utilización frecuente de fenitoina (datos no mostrados), generó una gran confusión clínica para adscribir esta reacción a un compuesto en particular.

El uso de corticoesteroides en esta patología continúa siendo polémico debido a que no existen reportes randomizados que avalen su eficacia en este contexto, y a las aprensiones derivadas de estudios en modelos animales que señalan la persistencia de microorganismos al interior del absceso a pesar del uso de $\operatorname{antibacterianos}^{25}$. Casi la mitad de los pacientes en esta serie recibió estos compuestos $(43,3 \%)$. Los que respaldan su uso señalan que forma parte de las estrategias para disminuir efectivamente la hipertensión endocraneana secundaria al edema vasogénico que rodea una lesión abscedada o tumoral. Visto así, los corticosteroides podrían ser utilizados en la fase aguda de la enfermedad para el manejo de una hipertensión endocraneana ${ }^{16}$.

Respuesta al tratamiento. El fracaso del tratamiento médico-quirúrgico fue frecuente en nuestros pacientes, alcanzando al 40\%, y que incluyó decesos, intervenciones quirúrgicas a pesar de un tratamiento médico inicial, reintervenciones o ajustes de esquema antimicrobiano. Estas cifras destacan la naturaleza dinámica de esta enfermedad y la posibilidad de optimizar algunos aspectos de manejo, como por ejemplo el momento quirúrgico y la selección de pacientes para tratamiento médico. En este fracaso no se incluyeron las modificaciones de tratamiento por reacciones adversas. La literatura médica consultada en general no incluye una descripción detallada sobre el fracaso del tratamiento médico o quirúrgico, más allá de la tasa de letalidad y por ello no fue posible comparar nuestros resultados. Sólo pudimos encontrar una cita ocasional sobre reacciones adversas en pacientes tratados con cloranfenicol $^{24}$.

Pronóstico. La incorporación de modernas técnicas imagenológicas, el desarrollo de técnicas quirúrgicas y el advenimiento de antimicrobianos con mejor actividad y penetración al SNC, ha modificado sustantivamente el pronóstico de esta enfermedad. La letalidad en nuestra serie es similar a la registrada en otras series contemporáneas $(\sim 10 \%)$ y notoriamente inferior a las cifras cercanas o superiores al $40 \%$, señaladas en la serie previamente publicada por este mismo hospital o por otros autores hacia 1980-19901-3,20,24. En el trabajo de Donoso et al, casi la mitad de los 42 pacientes reportados fueron identificados por informes de autopsia ${ }^{1}$. Una serie española reciente demuestra una caída en la letalidad de 33 a $5,4 \%$ en dos décadas ${ }^{17}$. La letalidad en niños en nuestro país también es baja ${ }^{3}$.

Los factores ligados a un mal pronóstico incluyen signos de herniación transtentorial al ingreso, rotura del absceso y lesiones múltiples ${ }^{3,18}$. En nuestra serie, aproximadamente un tercio de los pacientes fue tratado en forma médica con una letalidad similar a la del grupo quirúrgico, señalando que un subgrupo de pacientes puede ser seleccionado para esta modalidad terapéutica.

Secuelas y seguimiento. Las secuelas clásicas del absceso cerebral incluyen epilepsia, déficit intelectual y déficit neurológico. Nielsen et al, reportaron $30 \%$ de pacientes con secuelas de este tipo durante un seguimiento de varios años, una cifra cercana al $25 \%$ observado en esta serie ${ }^{26}$. Sin embargo, nuestros datos están sesgados por la limitada ventana de seguimiento disponible, por la falta de estandarización para medir 
estos eventos y porque algunas complicaciones, tales como la epilepsia, aparecen después de varios años ${ }^{26}$.

Aproximadamente, $50 \%$ de los pacientes desarrolla epilepsia durante o después del evento inicial. Los factores de riesgo asociados a esta condición incluyen absceso temporal o frontal y/o convulsiones durante el cuadro clínico ${ }^{26,27}$. La aparición de nuevas crisis convulsivas se concentra en los primeros 2 a 4 años post-egreso ${ }^{26,27}$.

En nuestra serie se registró otro deceso post-egreso asociado a la patología de base y re-hospitalizaciones por causas relacionadas, reflejando la morbilidad prolongada de esta patología. Excluyendo a la epilepsia, la curva de sobrevida libre de eventos sugiere que la evolución del paciente se estabiliza aproximadamente a los 4 meses de evolución.

Limitaciones. Es posible que no todos los pacientes pudieran haber sido detectados mediante la búsqueda por diagnóstico de egreso. Al menos un caso de absceso por hongo filamentoso en un paciente inmunocompetente, previamente publicado por nuestro grupo, no apareció en el listado de diagnósticos de egreso $^{28}$. También pudo haber ocurrido una exclusión obligada de pacientes graves con diagnóstico no confirmado por métodos imagenológicos y que pudo haber sesgado los cálculos de letalidad por esta patología. De la misma manera, los resultados aparentemente similares obtenidos con diferentes esquemas antimicrobianos, en presencia o ausencia de intervenciones quirúrgicas, no obedecen a una randomización y por lo tanto, no permiten responder la pregunta de cuál esquema es mejor o si es siempre necesario un tratamiento quirúrgico. No obstante, están en concordancia con la experiencia médica mundial que ratifica la importancia de un manejo multidisciplinario, con una selección caso a caso de la mejor opción terapéutica médica y quirúrgica. Este trabajo, tampoco exploró (por su diseño), la importancia del tratamiento anti-convulsivante profiláctico o la eficacia de las medidas anti-edema en el manejo de esta patología. Finalmente, a pesar de incluir 16 años de estudio, la reducida serie de pacientes, limita la exploración de asociaciones estadísticamente significativas por el bajo tamaño de la muestra.

\section{Resumen}

Se diseñó un estudio para conocer las principales características clínicas en 30 pacientes atendidos por absceso cerebral entre 1989 y 2005 . La puerta de entrada predominante fue por contigüidad (40\%) y luego inoculación directa por neurocirugía-trauma o diseminación hematógena $(23,3 \%)$.

La fiebre, cefalea y el compromiso de conciencia fueron las manifestaciones cardinales y la localización predominante fue temporal o frontal. Se observó un predominio de infecciones polimicrobianas y por cocáceas gram positivas.

La mayor parte fue sometida a tratamiento quirúrgico $(66,7 \%)$ y el resto a tratamiento médico solamente. Durante el tratamiento antimicrobiano, 6 pacientes $(20 \%)$ presentaron una reacción adversa que obligó a su suspensión o a cambio.

Se observó fracaso del tratamiento médico-quirúrgico en $40 \%$ de los casos, incluyendo 3 decesos $(10 \%$ letalidad), drenaje quirúrgico no programado o reintervenciones $(16,7 \%)$ o ajustes de esquema $(13,3 \%)$. Ocho pacientes presentaron secuelas al alta $(26,7 \%)$ y durante el seguimiento, $21 \%$ presentó rehospitalizaciones relacionadas o falleció.

\section{Referencias}

1.- Donoso A, Fernández M, Yánez A. Absceso cerebral: experiencia de 42 casos. Rev Méd Chile 1982; 110: 666-70.

2.- Olea P, Rivadeneira S. Absceso cerebral: análisis de 28 casos. Rev Chil Infect 1988; 5: 19-23.

3.- Podestá A. Lesiones inflamatorias nodulares encefálicas en el niño (Abscesosmicroabscesos-granulomas-tuberculomas). Rev Chil Neurocir 1987: 1: 41-51.

4.- Calfee DP, Wispelwey B. Brain abscess. Semin Neurol 2000; 20: 353-60.

5.- Heilpern K L, Lorber B. Focal intracranial infections. Infect Dis Clin North Am 1996; 10: 879-98.
6.- Sennaroglu L, Sozeri B. Otogenic brain abscess; review of 41 cases. Otolaryngol Head Neck Surg 2000; 123: 751-5.

7.- Viada J, Nazer A. Absceso cerebeloso secundario a otitis media crónica: caso clínico. Rev Otorrinolaringol Cir Cabeza Cuello 2000; 62: 271-6.

8.- Younis R T, Lazar R H, Anand V K. Intracranial complications of sinusitis: a 15 -year review of 39 cases. Ear Nose Throat J 2002; 81: 636-8, 640-2, 644

9.- Holzer F, Holzer J, Poblete R, Cornejo J. Infección postoperatoria en neurocirugía. Rev Chil Neurocir 1991; 5: 20-2.

10.- Pruitt AA, Rubin RH, Karchmer AW, Duncan GW. Neurologic complications of bacterial endocarditis. Medicine (Baltimore)
1978; 57: 329-43.

11.- Swanson K L, Prakash U B S, Stanton A W. Pulmonary arteriovenous fistulas: Mayo Clinic Experience, 1982-1997. Mayo Clinic Proc 1999; 74: 671-80.

12.- Enzmann DR, Britt RH, Placone R. Staging of human brain abscess by computed tomography. Radiology 1983; 146: 703-8.

13.- Mathisen, GE, Johnson JP. Brain abscess. Clin Infect Dis 1997; 25: 763-81.

14. - Lai P H, Ho J T, Chen W L, Hsu S S, Wang J S, Pan H B, et al. Brain abscess and necrotic brain tumor: Discrimination with proton $\mathrm{MR}$ spectroscopy and diffusion-weighted imaging. Am J Neuroradiol 2002; 23: 1369-77.

15.- Desprechins B, Stadnik T, Koerts G, Shabana W, Breucq C, Osteaux M. Use of diffusion- 
weighted MR imaging in differential diagnosis between intracerebral necrotic tumors and cerebral abscesses. Am J Neuroradiol 1999; 20: 1252-7.

16.- Korinek A M. Abscess and empyema of the brain. Rev Chil Neurocir 1994; 8: 22-7.

17.- González-García J, Gelabert M, Pravos AG, Villa JMF. Colecciones purulentas intracraneales. Revisión de 100 casos. Rev Neurol 1999; 29: 416-24.

18.- Yang SY. Brain abscess: a review of 400 cases. J Neurosurg 1981; 55: 794-99.

19.- Le Moal G, Landron C, Grollier G, Bataille B, Roblot F, Nassans P, et al. Characteristics of brain abscess with isolation of anaerobic bacteria. Scand J Infect Dis 2003; 35: 31821.

20.- Roche M, Humpreys H, Smyth E, Phillips J, Cunney R, McNamara E, et al. A twelveyear review of central nervous system bacterial abscesses; presentation and aetiology. Clin Microbiol Infect 2003: 9: 803-9.

21.- Fica A, Bozán F, Aristegui M, Bustos P. Espondilodiscitis. Análisis de una serie de 25 casos. Rev Méd Chile 2003; 131: 473-82.

22.- Briceño I, Pérez C, Jara A, Campos M, Troncoso P. Tratamiento exitoso de abscesos cerebrales múltiples por Nocardia en paciente sometido a trasplante renal. Rev Chil Infect 1998; 15: 52-6.

23.- Ferrer S, Baró M, Cárdenas M, Cartier L. Absceso cerebral por Toxoplasma gondii y SIDA: a propósito de un caso con estudio anatomo-patológico. Rev Méd Chile 1993; 121: 1036-41.

24.- Schoihet S, De Ramón R. Abscesos intracraneanos por hongos a propósito de un caso de aspergillosis cerebral. Rev Chil Neurocir 1991; 5: 54-7.
25.- Quartey G R, Johnston J A, Rozdilsky B. Decadron in the treatment of cerebral abscess. An experimental study. J Neurosurg 1976; 45: 301-10.

26.- Chun C H, Johnson J D, Hofstetter M, Raff M J. Brain abscess. A study of 45 consecutive cases. Medicine (Baltimore) 1986; 65: 415-31.

27.- Nielsen H, Harmsen A, Gyldensted C. Cerebral abscess. A long-term follow-up. Acta Neurol Scand 1983; 67: 330-7.

28.- Legg N J, Gupta P C, Scott D F. Epilepsy following cerebral abscess. A clinical and EEG study of 70 patients. Brain 1973; 96 : 259-68.

29.- Fica A, Díaz M C, Luppi M, Olivares R, Saez L, Baboor M, et al. Unsuccessfull treatment with voriconazol of a brain abscess due to Cladiophialophora bantiana. Scan J Infect Dis 2003; 35: 892-93. 